\title{
East Indian Lemongrass Oil
}

National Cancer Institute

\section{Source}

National Cancer Institute. East Indian Lemongrass Oil. NCI Thesaurus. Code C107304.

The essential oil of Cymbopogon flexuosus. East Indian lemong rass oil has antifungal activity. 